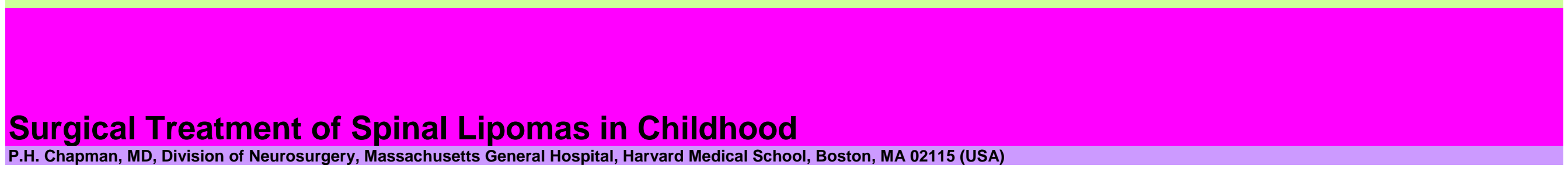

\title{
Introduction
}

Spinal lipomas presenting as a soft subcutaneous mass in the lumbosacral region should cause concern regarding possible neurologic implications. These lesions are present a birth and their rate of increase in size is proportional to body growth in general. They usually consist of a variety of tissues besides fat, including muscle, bone, and even enteric elements (fig. 1) [1]. With rare exceptions, the subcutaneous mass extends intraspinally and attaches in some manner to the spinal cord. There is variable involvement of nerve roots of the cauda equina. The affected spinal cord is elongated, continuing well down into the lumbar or even sacral canal. The normal conus terminates in the region of T-12 to L-2 and should not extend below the T2-3 interspace [2]. Elongation and tethering of the conus are features shared by other types of occult spinal dysraphism besides spinal lipomas.

It has been recognized for some time that lipomas are associated with lower extremity sensorimotor deficits as well as bladder and bowel disturbance [3-5]. These deficits are usually relatively minor in early life in contrast to the severe abnormalities commonly occurring with my-elomeningocele. In infants with lipomas and neurologic abnormalities, disordered bladder function is particularly common. The more serious concern, however, is neurologic deterioration at any time throughout childhood and even in adult life. Often the patient has been normal up to

that point in spite of which progressive deficits of frightening degree may ensue. Such a circumstance naturally lends itself to attempts at preventing the problem.

As with other forms of occult spinal dysraphism with cord tethering, the goal of treatment is to release conus from the mechanical constraint which the lipoma presents. In the simplest circumstance there is a stout fibrolipomatous stalk which continues through a posterior dural defect to attach to the distal conus. In this instance, untethering is achieved by merely dividing the stalk. Much more commonly, however, the lipoma has a substantial interface with the spinal cord. In addition, nerve roots of the cauda equina are intimately related to the mass. In order to effectively and safely untether the cord in such cases, an appreciation of the surgical anatomy is important. Until recently there has been a paucity of surgically useful anatomic information. The important study of Emery and Lendon [6], which emphasized the nature of leptomyelolipomas, is of limited help to the surgeon in dealing with these difficult lesions. Naidich et al. [7] have discussed the place of preoperative studies in planning a surgical approach. We have recently described a classification of spinal lipomas predicated on their anatomy as observed at surgery [8]. A method of surgical approach was present based on that classification. The purpose of this paper is to further amplify our findings correlating x-ray studies, operative experience, and histo-logic material.

Thanks are due to Annette Lehmann for her assistance with the preparation of this manuscript.

(C) 1993 S.KargerAG, Basel

$1016-2291 / 93 / 0195-0267$

$\$ 2.75 / 0$ 

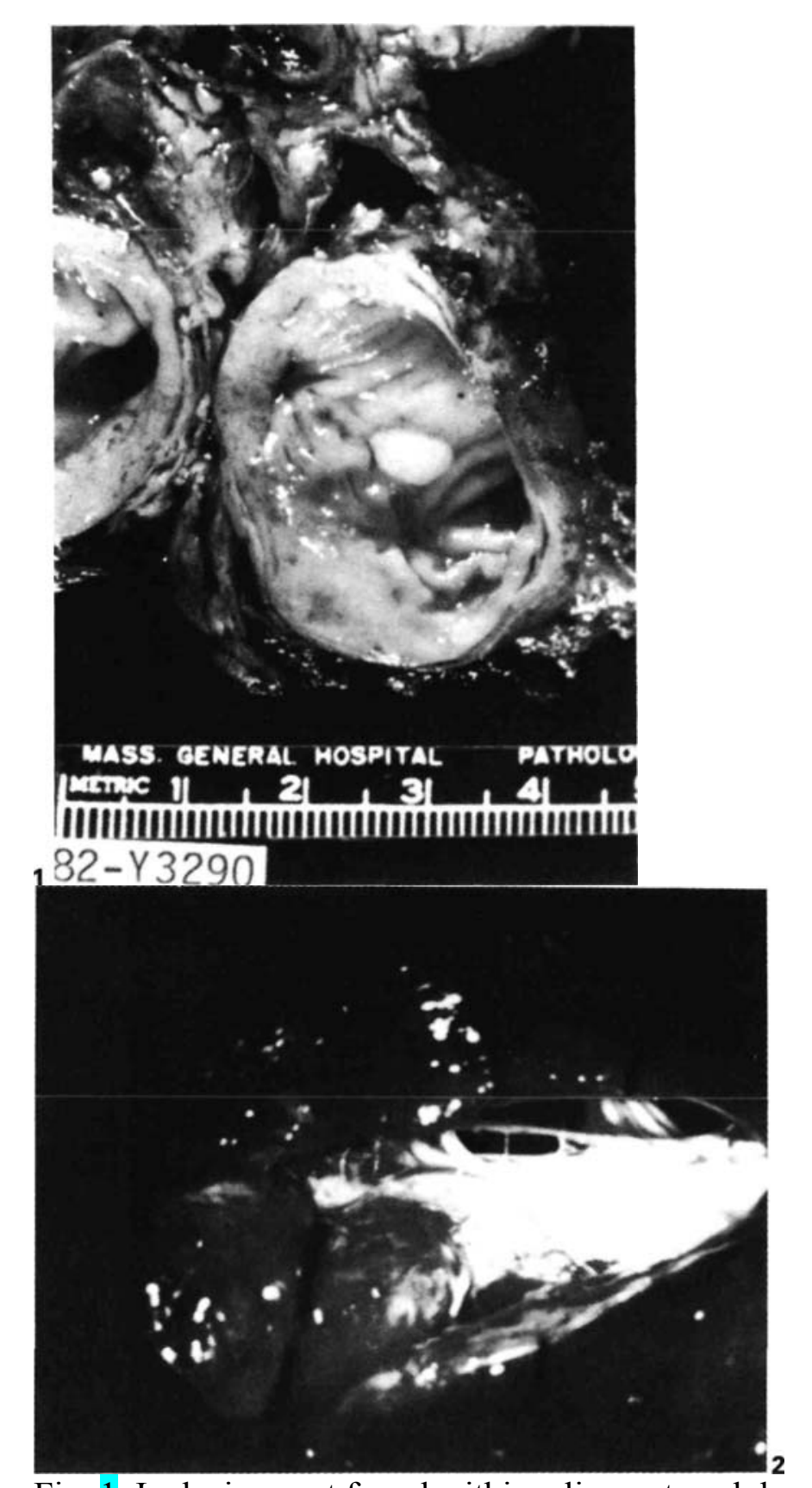

Fig. 1. Inclusion cyst found within a lipomatous lubosacral mass in an infant. Gastric musosa, muscularis layers, and serosa constitute the cyst wall. A chronic 'gastric' ulcer is present.

Fig. 2. Intradural exposure of dorsal lipoma showing the relationship of posterior nerve roots to the lipoma-cord interface (upper).

Method and Results

Surgical Anatomy and Approach

The interface between lipoma and conus is both extensive and poorly demarcated with gradual merging of neural and fibrolipomatous elements. It is therefore essential to remain oriented as to the location of neural structures while detaching the lipoma. Two anatomic features useful in planning an operative approach and maintaining orientation during surgery are: (1) the site of attachment of the lipoma to the conus and (2) the position of posterior nerve roots in relation to this lipoma-cord interface. At its two extremes the lipoma may attach either to the posterior aspect of the conus or to its caudal end. In addition, however, there are intermediate forms which represent a continuum between these two extremes. We shall briefly consider each of these types in turn, first dorsal, then caudal, and, finally, intermediate.

In the case of lipomas which insert on the dorsal aspect of the conus, one finds that posterior nerve roots emerge on both sides just ventral to the lipoma-cord interface. Moreover, the external aspect of this interface corresponds closely to the circumferential limits of the congeni-

tal dural defect which the lipoma penetrates as it enters the spinal canal. The edges of this dural defect merge with the lipoma-cord junction, creating a line of fusion between lipoma, cord and dura. If one merely transects the lipoma at the level of the posterior dura, the persisting attachment between dura and cord prevents satisfactory untethering. In order to effectively release the conus one must completely divide this attachment. Because posterior nerve roots emerge just anterior to the line of fusion, they provide a useful orienting landmark. Initially, the dura is opened rostral to the lipoma in order to identify the conus. The opening is then carried caudally on either side of the lipoma to identify posterior roots (fig. 2). The lipoma is progressively transected, freeing it from the posterior aspect of the conus. At the same time the lateral line of attachment between dura and cord-lipoma interface is divided circumferentially (fig. 3). All dissection is performed in a plane just dorsal to the posterior roots in order to avoid damage to neural structures.

The lipoma may attach to the distal extremity of the conus. In such cases, the elongated conus expands to merge with a more caudal fibrofatty intraspinal mass. This mass, in turn, penetrates a dural defect at the end of 

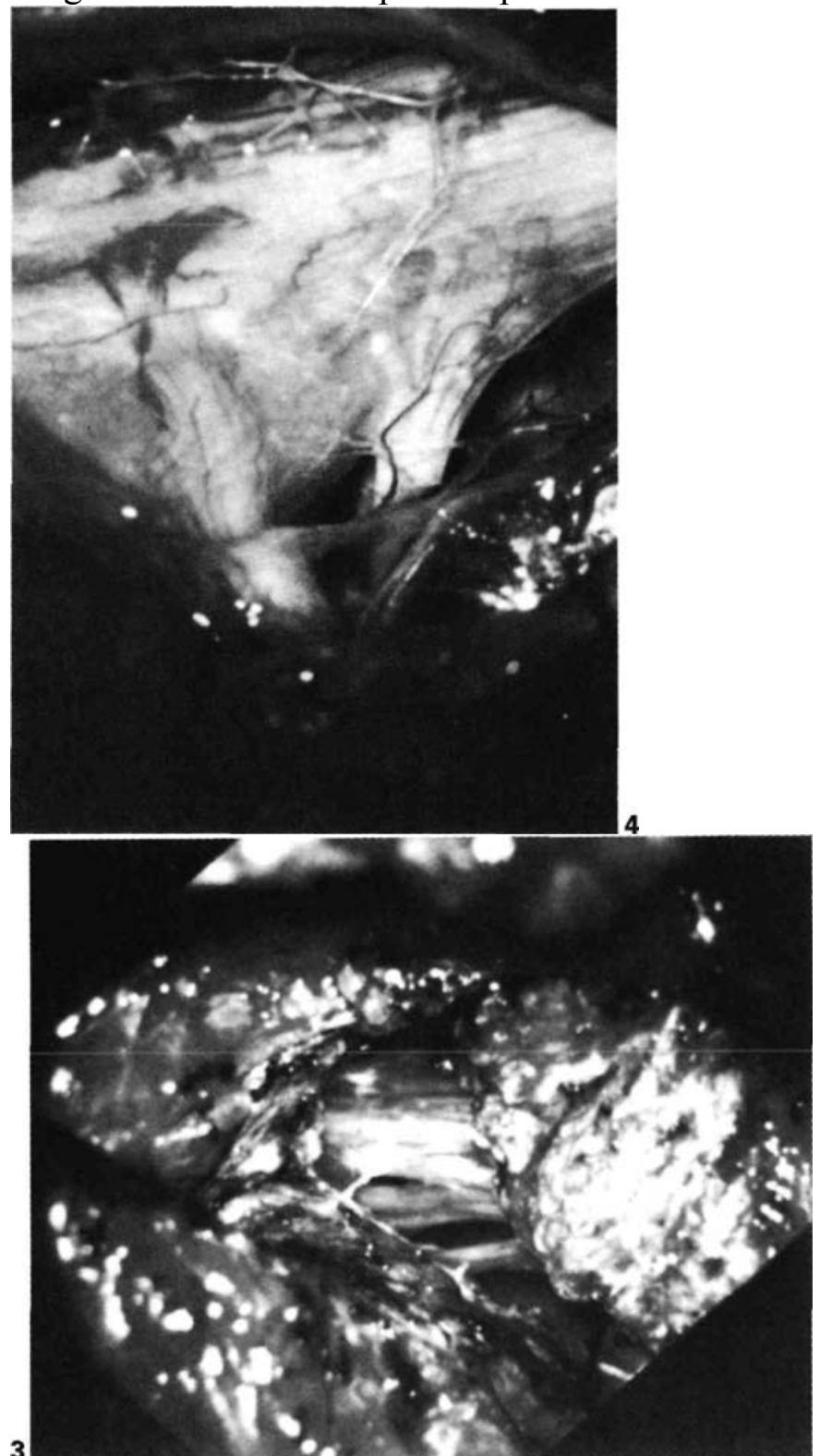

Fig. 3. Distal extent of dorsal lipoma following circumferential division of dural attachments to the lipoma-cord interface. Note the emerging nerve roots ventral to the line of detachment.

Fig. 4. Caudal lipoma in the region of the lipoma-cord interface. Note the posterior nerve root emerging dorsal to the lipoma tissue.

the spinal canal. It may present as a subcutaneous lipoma or be imperceptible clinically. The caudal lipomas differ from the dorsal in two important respects. First, although the edges of the dural defect are fused to the penetrating lipoma, the attachment is not in proximity to the lipoma-cord interface but appears to lie caudal to this. Therefore, one finds a substantial mass of lipoma which seems to lie within the dural cul-de-sac. Second, posterior roots do not reliably define the site of the lipoma-cord junction (fig. 4). In the case of caudal lipomas, one may achieve untether-ing by dividing the lipoma distal to its junction with the conus without having to contend with a tethering dural attachment at the interface. It is essential, however, to be sure that the level of transection is beyond any functionally important neural elements. This can be done in a variety of ways. The simplest is to monitor bladder and lower extremity motor responses to nerve stimulation. Rectal electromyographic recording has been used as well. Soma-tosensory-evoked responses, though more elaborate, have the advantage of identifying sensory elements. One typically encounters small nerves within the substance of the lipoma, particularly in its more ventral portions. Histo-logic examination shows a disorganized cross-sectional anatomy with fat, fibrous tissue and skeletal muscle interspersed among myelinated and unmyelinated nerve fibers, glial islands, ganglion cells, and ependymal rests

(fig. 5). In order to achieve untethering, it is necessary to divide such nerve-bearing tissue. The importance of properly identifying and sparing significant neural elements by the means described above is emphasized.

Although the dorsal and caudal lipomas represent polar types, there are intermediate forms representing a continuum between the two. It is typical of these intermediate lesions that the rostral portion resembles the dorsal form, while distally they are similar to the caudal lipomas. This means that rostrally one can expect the line of fusion between the dura and lipoma-cord complex to lie posterior to emerging sensory rootlets. As one proceeds caudally, however, there is a zone of transition wherein the line of fusion between the lateral dura, cord and lipoma has migrated anteriorly, so that the posterior nerve roots begin to emerge from the lateral surface of the lipoma. Here, one should initially transect the lipoma posterior to these roots. Thereafter, one can dissect laterally through the lipoma to divide the dural attachment which may lie in the anterolateral or even anterior spinal canal. In doing this, the roots traversing and emerging from the 
lipoma should be spared. Finally, the remaining lipoma can be transected at its caudal extremity. Again, nerve stimulation is essential to identify and spare functional neural elements.

269

V

31

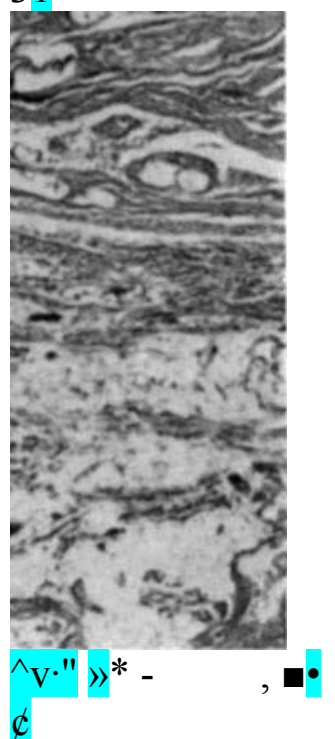

Fig. 5. Histologic cross-section of a caudal lipoma at the level of transection. Small nerve fibers are scattered randomly in a matrix of abundant fibrous tissue, fat, and skeletal muscle. An ependyma-lined canal (large arrow upper left) and an island of glia (small arrows) are present.

Radiologic Studies

Given the variable anatomic configuration of lipomas and the corresponding variations in surgical approach, it is desirable to have complete and accurate $\mathrm{x}$-ray studies preoperatively. Metrizamide myelography with polyto-mography has begun to supplant air myelography. Recently, computerized body tomography (CBT) has demonstrated its usefulness. Metrizamide or air is introduced by cisternal or lateral cervical puncture for myelography. Anteroposterior and lateral tomographic views are obtained. When using positive contrast, this may be followed by axial CBT views. In the case of younger children, it is possible to obtain on-line anteroposterior and lateral CBT projections with intrathecal metrizamide, obviating the need for preliminary myelography (fig. 6). The threedimensional representations obtained with these studies allow one to predict with considerable accuracy the type of lipoma in question.

Myelographically, dorsal lipomas present as a symmetrical expansion of the elongated conus which is displaced posteriorly and is confluent with adjacent dura over a considerable extent corresponding to the congenital dural defect (fig. 7). The thecal sac extends caudally from the level. Axial

CBT views confirm the symmetrical disposition of the conus and the attached lipoma. This view also demonstrates the posterolateral limits of the dura at its point of attachment to the conus-lipoma interface on each side (fig. 8). This allows one to anticipate the extent of lateral dissection necessary in order to release the lateral dural attachment and to predict the position of posterior nerve roots.

Caudal lipomas are seen as a fusiform expansion of the elongated conus usually with a bulbous termination which obliterates the dural cul-de-sac (fig. 9). Cross-sectional views show progressive replacement of the conus by lipoma (fig. 10). The location and extent of any posterior or lateral dural defect and its level can be ascertained by these views. Intermediate lipomas exhibit an admixture of these findings, as one might expect. Rostrally, there is expansion and posterior displacement of the conus, so that it becomes confluent with the overlying dura. Caudally, there is further expansion of the mass to fill the thecal culde-sac (fig. 6). Axial views through the lesion are quite helpful in defining the degree of asymmetry of the dural attachment (fig. 11). As described above, the line of dural fusion follows the lipoma-cord interface and therefore allows one to anticipate its location at any level.

270

Chapman/Davis

Surgical Treatment of Spinal Lipomas in Childhood

Fig. 6. Anteroposterior and lateral CBT views following instillation of intrathecal metrizamide. An intermediate type of lipoma is shown. 


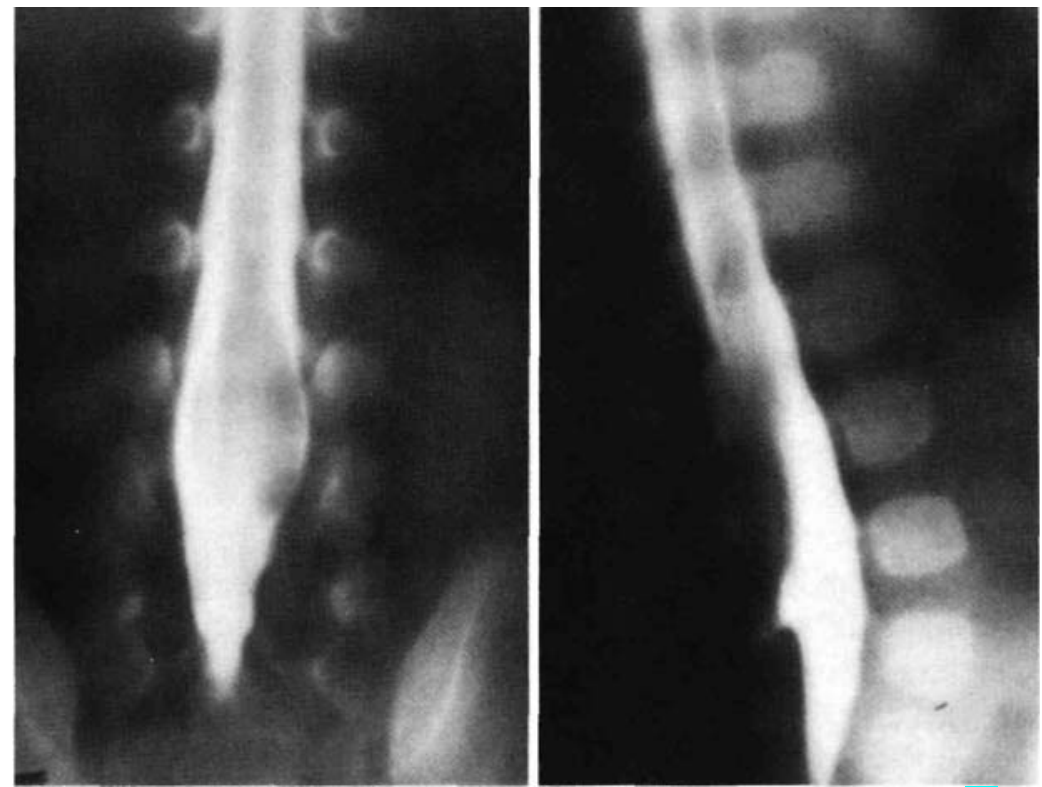

Fig. 7. Anteroposterior and lateral metrizamide myelogram showing the dorsal form of lipoma.

Clinical Experience

Using the approach described, we have now operated on 17 children and adolescents with lipomas, 13 of whom were female and 4 male. Their ages ranged from 5 days to 17 years at the time of surgery. Associated anomalies included 2 cases of bladder extrophy, one with imperfo-rate anus and another with sacral agenesis. 2 children had

occipital encephaloceles in infancy and a 3rd had partial sactal agenesis. No case of Arnold-Chiari malformation was found. 5 children had neurogenic

bladders in early infancy, 1 with distal leg weakness. Both children with bladder extrophy were also born with distal leg weakness. Of the 7 children with birth or early infancy deficits, 5 developed further problems of weakness, bladder distur-

271

Fig. 8. Axial CBT of dorsal lipoma with intrathecal metrizamide.Spinal cord and attached lipoma lie within the posterior portion (up- Fig. 9. Caudal lipoma with characteristic obliteration of the the-

per) of the metrizamide-filled thecal sac. cal cul-de-sac.
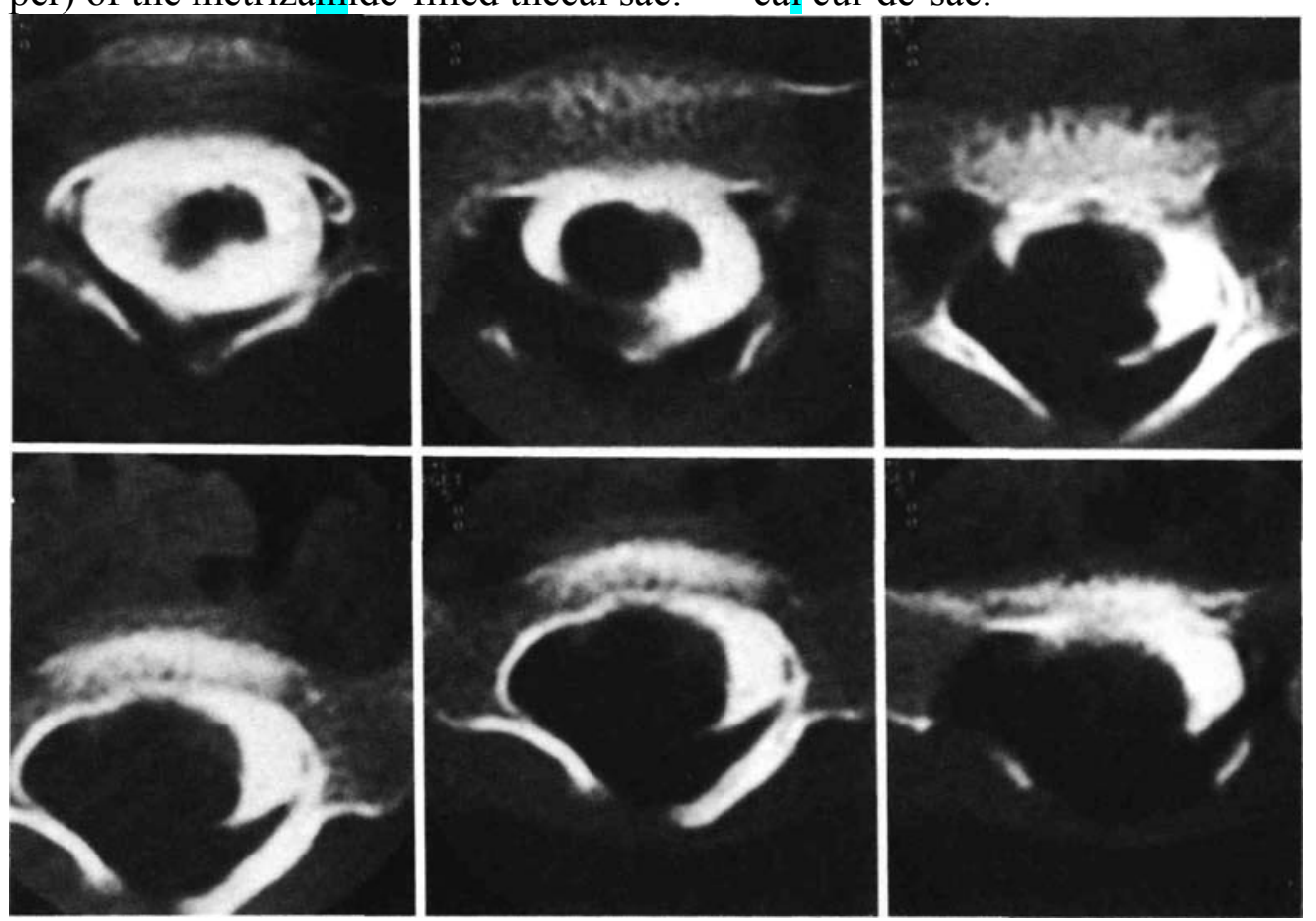

Fig. 10. Seria $1 / 8 x i a l$ CBT views of a predominantly caudal lipoma. This shows progressive transition from the conus proximally (upper left) to the lipoma distally (lower right). The patient's posterior aspect is oriented toward the lower portion of each figure.

272

Chapman/Davis

Surgical Treatment of Spinal Lipomas in Childhood 


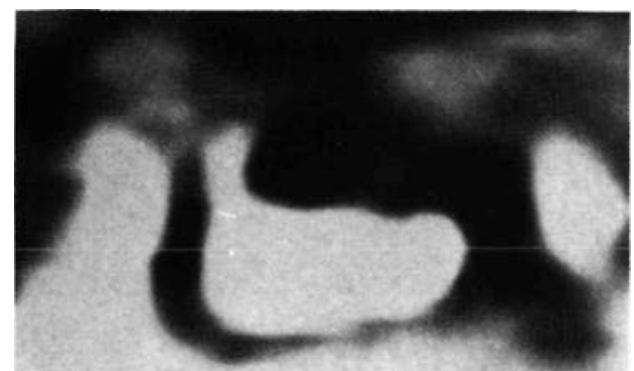

Fig. 11. Axial view of an intermediate form of lipoma. Note the asymmetry of this lesion with displacement of the line of dural fusion ventrally (lower) on the right.

bance, sensory loss, or pain. There were 6 patients, normal in early life, in whom delayed deficits occurred. Thus, a total of 11 out of the 17 patients exhibited postnatal neurologic deterioration which occurred from ages 3 months to 16 years. 4 neurologically normal infants were operated upon between 5 days and 6 months of age, the indication for surgery being the presence of the lipoma itself.

On the basis of radiographic and surgical finding, we classified 4 of the lipomas as dorsal, 6 as caudal, and 7 intermediate. Satisfactory untethering was possible except in 1 patient who had two prior attempts at removal with resulting severe arachnoiditis. Postoperatively, there was 1 case of transient cerebrospinal fluid leak, but no other morbidity. No child exhibited neurologic worsening as a result of surgery, although 3 patients with progressive pes cavus deformities preoperatively had some further worsening of this problem in spite of surgery. The remainder who had deteriorated before surgery, stabilized at that level of function.

Discussion

One aspect of prevention is being able to predict the likelihood of a problem occurring. There are no available prospective data which allow one to anticipate the probability of deterioration in a given child with spinal lipoma. On the other hand, observations made in the cases of children followed without treatment for one reason or anoth-

er suggest an incidence of deterioration as high as $80 \%$ [4]. In our own experience, 4 out of 5 children having earlier excision of the subcutaneous mass alone subsequently developed serious progressive deficits [8]. These observations, combined with the common history of deterioration in most series, compel serious concern regarding the nonoperative treatment of spinal lipomas. Obviously, however, prevention implies an effective treatment. As noted, simple decompression by removal of the extradu-ral portion of the mass has no effect on later deterioration. To be useful, surgery must be directed at untethering the conus from its attachments to the lipoma. It is the tethering effect, possibly causing impaired spinal cord oxidative metabolism via ischemia, which is the important pathologic factor [9]. This is, or course, similar to other types of occult dysraphism such as diastomatomyelia, thickened filum terminale, or meningocoele manque. In the case of lipomas, however, one is often confronted with a complex anatomic relationship between lipoma and neural elements which make attempts at untethering not only confusing but hazardous.

Our concern has been to develop a method of approach to spinal lipomas which allows effective and safe untethering of the conus. This approach is based on what we perceive to be their surgically pertinent anatomy. The classification into dorsal, caudal, and intermediate forms is strictly observational and is not meant to have embryo-logical implications. The usefulness of this classification lies in allowing one to anticipate the location of neural structures in relationship to lipoma and to tailor one's surgical approach accordingly. This depends in turn on accurate preoperative radiographic studies.

In our experience, the intermediate type of lipoma has been the most difficult to approach surgically. This reflects its transitional nature wherein dorsal roots no longer provide a reliable landmark for safe dissection, and the interface betwen lipoma, conus and dura migrates ventrally, leaving nerve elements to traverse the fatty mass. In addition, there is usually asymmetry between the two sides which can cause a problem of orientation. When the transitional zone is properly identified, however, dissection can be modified to deal with the more caudal portion of the mass. We feel that the treatment of lipomas is best carried out in infancy or early childhood, prior to the onset of progressive sensory, motor, or sphincter problems. This also takes advantage of the greater technical ease of operation at that time. In adolescence, the exposure of an extensive lipoma, using microsurgical techniques, may be tedious, reflecting the volume and fibro-vascular consistency of the tissue.

273

References

Walsh JW, Markesbery WR: Histological features of congenital lipomas of the lower spinal canal.J/Neurosurg 1980;52:564-569. Barson AJ: Vertebral level of termination of spinal cord during normal and abnormal development. JAnat 1969; 106:489-497. Bassett RC: The neurologic deficit associated with lipomas of the cauda equina. Ann Surg !95O;131:109-116.

Bruce DA, Schut L: Spinal lipomas in infancy and childhood. Childs Brain 1979;5:192-203. Swanson HS, Barnett JC: Intradural lipomas in children.

Pediatrics 1962;29:91 1-926. Emery JC, Lendon RG: Lipomas of the cauda equina and other fatty tumours related to neu-rospinal dysraphism. Dev Med Child Neurol 1969;20(suppl):62-70.

Naidich TP, McLone DG, Palacio CA: Presur-gical evaluation of lipomyelomeningocoeles. Annu Meet Am Soc Neuroradiol, Chicago, 1981.

Chapman PH: Congenital intraspinal lipomas. Anatomic considerations and surgical treatment. Childs Brain 1982;9:37-47. Yamada S, Zinke DE, Sanders D: Pathophysi-ology of tethered cord syndrome'. J Neurosurg 1981:54:494-503.

\section{P.H. Chapman}

Author's Comment

Over the past 15 years, operative treatment for spinal lipomas has become standard, and pediatric neurosurgi-cal units around the world have accumulated substantial experience with these difficult lesions [1-4]. Newer, non-invasive imaging modalities have simplified the problem of diagnosis. Ultrasonography is 
useful for screening suspected cases of occult dysraphism in neonates [5]. In some instances, MRI provides images that are comparable to those obtained by CT myelography. MRI is also useful for detecting the presence of hydromyelia, especially with myelocystocele, which is typically associated with a large, fatty subcutaneous mass [6]. However, the anatomic resolution that MRI provides may not be adequate for operative planning [7,8]. This is particularly true for studies done on infants and younger children, when a transitional lipoma is present, or after surgery has already been performed.

Urologic abnormalities are common in children with lipomas, even in the absence of overt symptoms or other neurologic deficits [9]. For this reason, preoperative urologic assessment with urodynamics is appropriate. Likewise, intraoperative monitoring should include segmental levels pertinent to bladder and bowel function. Various methods have been suggested to accomplish this, including bladder and rectal manometry, detrusor/sphincter EMG recordings, and sensory evoked potentials. WE monitor perineal and lower extremity EMG responses to bipolar stimulation during dissection and have found this to be as reliable and simple as any other method.

In some cases of spinal lipoma, the large volume of fat may present a technical problem. It can cause neurologic deficits by mass effect. During operative dissection, it also obscures normal anatomic structures and represents an unacceptable bulk of tissue to be left in the spinal canal after untethering. The $\mathrm{CO} 2$ laser is quite helpful for expeditious removal of fat. One must, however, use it with caution when there are functionally important neural elements within the fatty mass. This can occur with transitional lipomas or caudal lipomas where the conus-lipoma interface is sufficiently rostral, i.e. above the lumbosacral junction.

Retethering is a significant cause of late postoperative morbidity. Various surgical maneuvers have been devised in an effort to reduce the incidence of this complication, although it continues to be about $10 \%$ in all series [2]. In my experience, retethering is more likely to occur with complex, transitional lipomas which have an extensive lateral (or even anterolateral) attachment. In these cases, adequate repair of the dural defect may be a problem, leaving raw tissue surfaces in close juxtaposition where they may more easily adhere to each other. The nerve roots themselves may be short and dysplastic, limiting the degree to which the conus can be mobilized [10]. The diagnosis of symptomatic retethering is also a problem area. Up to now, there has been no single, reliable test for retethering. MRI and myeolography will often give the appearance of persistent/recurrent tethering, even in the absence of symptoms or change in the neurologic exam.

\section{Chapman/Davis Surgical Treatment of Spinal Lipomas in}

Childhood

Consequently, one must rely heavily on one's own experience and clinical judgement in assessing the significance of equivocal symptoms and weighing the need for further surgery. Recently, McCuUough et al. [11] have reported on the use of cervical spinal cord motion measurements

by MRI scanning to detect tethering of the conus medul-laris. If their experience is corroborated by others, we will have a very useful diagnostic tool to reliably assess rete-thering and perhaps even refine our indications for initial surgery.

References

Kanev PM, Lemire RJ, Loeser JD, Berger MS: Management and long-term follow-up review of children with lipomyelomeningocele, $1952-1987$. JNeurosurg 1990;72:48-52. Sakamoto H, Hakuba A, Fujitani K, Nishimu-ra S: Surgical treatment of the retethered spinal cord after repair of lipomyelomeningocele. J Neurosurg 1991;74:709-714. Hoffman HJ, Taecholarn C, Hendrick EB, Humphreys RP: Management of lipomyelome-ningoceles: Experience at the Hospital for Sick Children, Toronto. J Neurosurg 1985;62:1-8. Pierre-Kahn A, Lacombe J, Pichon J, et al: Intraspinal lipomas with spina bifida. Prognosis and treatment in 73 cases. J Neurosurg 1986:65:756-761.

Korsvik HE, Keller MS: Sonography of occult dysraphism in neonates and infants with MR imaging correlation. Radiographics 1992;12: 297-306. Cohen AR: The mermaid malformation: Cloa-cal exstrophy and occult spinal dysraphism. Neurosurgery 1991;28:834-843.

Brophy JD, Sutton LN, Zimmerman RA, Bury E, Schut L: Magnetic resonance imaging of lipomyelomeningocele and tethered cord. Neurosurgery 1989;25:336-340.

O'Neill P, Stack JP: Magnetic resonance imaging in the pre-operative assessment of closed spinal dysraphism in children. Pediatr Neurosurg 1990/1991;16:240-246.

9 Atala A, Bauer SB, Dyro FM, Shefner J, et al: Bladder functional changes resulting from lipomyelomeningocele repair. J Urol 1992;148: 592-594. Barolat G, Schaefer D, Zeme S: Recurrent spinal cord tethering by sacral nerve root following lipomyelomeningocele surgery. Case report. J Neurosurg 1991;75:143-145.

Articles of interest taken from the series of monographs entitled Concepts in Pediatric Neurosurgery are reproduced exactly as they appeared at the time of their publication. These papers represent the materials presented and discussed at past meetings of the American Society of Pediatric Neurosurgeons. The original article is followed by the senior author's commentary and update. 